\title{
Myo-inositol may prevent gestational diabetes onset in overweight women: a randomized, controlled trial
}

Angelo Santamaria, Antonino Di Benedetto, Elisabetta Petrella, Basilio Pintaudi, Francesco Corrado, Rosario D’Anna, Isabella Neri \& Fabio Facchinetti

To cite this article: Angelo Santamaria, Antonino Di Benedetto, Elisabetta Petrella, Basilio Pintaudi, Francesco Corrado, Rosario D'Anna, Isabella Neri \& Fabio Facchinetti (2016) Myoinositol may prevent gestational diabetes onset in overweight women: a randomized, controlled trial, The Journal of Maternal-Fetal \& Neonatal Medicine, 29:19, 3234-3237, DOI: 10.3109/14767058.2015.1121478

To link to this article: http://dx.doi.org/10.3109/14767058.2015.1121478

曲 Published online: 23 Dec 2015.

Submit your article to this journal $₫$

Џll Article views: 158

Q View related articles ¿

View Crossmark data $\widetilde{ }$ 


\title{
Myo-inositol may prevent gestational diabetes onset in overweight women: a randomized, controlled trial
}

\author{
Angelo Santamaria ${ }^{1}$, Antonino Di Benedetto ${ }^{2}$, Elisabetta Petrella ${ }^{3}$, Basilio Pintaudi ${ }^{2}$, Francesco Corrado ${ }^{1}$, \\ Rosario D'Anna ${ }^{1}$, Isabella Neri ${ }^{3}$, and Fabio Facchinetti ${ }^{3}$ \\ ${ }^{1}$ Department of Pediatrics, Gynecology, Microbiology and Biomedical Sciences, University of Messina, Messina, Italy, ${ }^{2}$ Department of Clinical and \\ Experimental Medicine, University of Messina, Messina, Italy, and ${ }^{3}$ Obstetric Unit, Mother-Infant Department, University of Modena and Reggio \\ Emilia, Modena, Italy
}

\begin{abstract}
Objective: To evaluate whether myo-inositol supplementation may reduce gestational diabetes mellitus (GDM) rate in overweight women.

Methods: In an open-label, randomized trial, myo-inositol ( $2 \mathrm{~g}$ plus $200 \mu \mathrm{g}$ folic acid twice a day) or placebo $(200 \mu \mathrm{g}$ folic acid twice a day) was administered from the first trimester to delivery in pregnant overweight non-obese women (pre-pregnancy body mass index $\geq 25$ and $<30 \mathrm{~kg} / \mathrm{m}^{2}$ ). The primary outcome was the incidence of GDM.

Results: From January 2012 to December 2014, 220 pregnant women were randomized at two Italian University hospitals, 110 to myo-inositol and 110 to placebo. The incidence of GDM was significantly lower in the myo-inositol group compared to the placebo group $(11.6 \%$ versus $27.4 \%$, respectively, $p=0.004$ ). Myo-inositol treatment was associated with a $67 \%$ risk reduction of developing GDM (OR 0.33; 95\% Cl 0.15-0.70).

Conclusions: Myo-inositol supplementation, administered since early pregnancy, reduces GDM incidence in overweight non-obese women.
\end{abstract}

\section{Keywords}

Body mass index, gestational diabetes, myo-inositol, obese, overweight

\section{History}

Received 11 October 2015

Revised 14 November 2015

Accepted 15 November 2015

Published online 16 December 2015

\section{Introduction}

Gestational diabetes mellitus (GDM) is defined as carbohydrate intolerance that begins or is first recognized during pregnancy [1]. It is associated with an increased risk for the mother and fetus [2]. From the beginning of 2010, after the publication of the Hyperglycemia and Adverse Pregnancy Outcomes (HAPO) study results [2], we follow the recommendations of the International Association of Diabetes in Pregnancy Study Group (IADPSG) [3] later modified in 2011 by American Diabetes Association (ADA) [4]. However, these new criteria increased the number of GDM diagnoses to more than double [5,6], especially in women at risk. In recent years, our group demonstrated that a diet supplement, an isomer of inositol, the myo-inositol, has insulin sensitizing effects [7] and may decrease GDM occurrence in populations at risk for GDM, like obese women [5] or women with family history for Diabetes Mellitus type 2 [6]. Myo-inositol is a cyclitol, normally present in cereals, corn, legumes and meat, but also synthesized by our body, principally in the liver. In many countries, myo-inositol is on the market as a

Address for correspondence: Angelo Santamaria, Department of Pediatrics, Gynecology, Microbiology and Biomedical Sciences, University of Messina, via C. Valeria, n. 1, 98100 Messina, Italy.

Tel: +39090 2217324. E-mail: angelosantamaria83@alice.it dietary supplement [8], despite its proven pharmacological effects [9]. BMI ranging 25.1-29.9 is considered a risk factor predisposing to adverse outcomes in pregnancy [10], such as GDM and its complications [11]. In this study, we specifically considered only overweight non-obese women, with the aim of exploring if also in this category of high risk women, myo-inositol supplementation might reduce GDM incidence.

\section{Methods}

A randomized, controlled, double-centre, open-label study was performed involving pregnant outpatients attending the Units of Gynecology and Obstetrics of two Italian University Hospitals located in Messina and Modena. The recruitment started at the beginning of 2012 and lasted 36 months until reaching the target inclusion of 220 pregnant women. The inclusion criteria were: (1) pre-pregnancy BMI $>25$ and $<30 \mathrm{~kg} / \mathrm{m}^{2}$, (2) first trimester fasting plasma glucose $\leq 126 \mathrm{mg} / \mathrm{dl}$ and/or random glycemia $<200 \mathrm{mg} / \mathrm{dl}$, (3) single pregnancy and (4) Caucasian ethnicity. Exclusion criteria were as follow: (1) pre-pregnancy BMI $<25$ and $\geq 30 \mathrm{~kg} / \mathrm{m}^{2}$, (2) previous GDM, (3) pre-gestational diabetes, (4) first trimester glicosuria and (5) treatment with corticosteroids. The main outcome measure was the occurrence of GDM. Secondary outcomes were prevalence of fetal macrosomia 
(fetal birth weight $>4000 \mathrm{~g}$ at delivery), rate of cesarean section, preterm delivery ( $<37$ weeks), Pregnancy Induced Hypertension (PIH). Cases of shoulder dystocia, neonatal hypoglycemia and neonates transferred to NICU (Neonatal Intensive Care Unit) were also recorded. According to ADA recommendations [4], a diagnosis of GDM was performed at 24th-28th week of gestation with a 75-g 2-h oral glucose tolerance test (OGTT), cut off values of $\geq 92 \mathrm{mg} / \mathrm{dl}$ fasting, $\geq 180 \mathrm{mg} / \mathrm{dl} 1$-h post-load and $\geq 153 \mathrm{mg} / \mathrm{dl}$ 2-h post-load; at least one of the three values that exceeds or equals the cut off was enough to diagnose GDM. At the time of the recruitment (12th-13th week), all the consecutive eligible women who accepted to participate, after provided written informed consent, were randomized to placebo or treatment group as reported below. Randomization list was obtained by using a computer-generated random allocation with a 1:1 ratio. The allocations were sealed in numbered white envelopes, which were kept in the midwifery facility. After the eligibility was assessed, a midwife opened the next random envelope. Because of the study design, the gynecologist knew the group allocation of the patient. Treated group received $2 \mathrm{~g}$ myo-inositol given twice a day plus $200 \mu \mathrm{g}$ folic acid, whereas to placebo group only $200 \mu \mathrm{g}$ folic acid twice a day was administered. Information on the occurrence of side effects caused by treatments was collected during follow-up visits. In particular, the presence of nausea, flatulence, diarrhea, headache, insomnia, uterine contractions and tiredness was assessed. At enrollment, fasting glucose and insulin levels were measured, in order to evaluate the Homeostasis Model Assessment-Insulin Resistance index (HOMA-IR). HOMAIR index was calculated as follows: [(fasting glucose $\mathrm{mg}$ / dl) $\times$ (fasting insulin $\mathrm{mUI} / \mathrm{l}) / 405$ ]. Serum insulin was measured through an ELISA commercial kit (DRG Diagnostics, Marburg, Germany), and the concentrations are expressed in $\mu \mathrm{IU} / \mathrm{ml}$. The protocol was consistent with the principles of the Declaration of Helsinki, and all participants gave their written informed consent. The trial is registered as number NCT01047982. The trial was approved by the Ethical Committee of Messina University Hospital. Taking into account that previous trials $[5,6]$ showed a reduction of $\sim 67 \%$ in the rate of GDM development in the group of women treated with myo-inositol with respect to placebo, and considering that the expected rate of GDM in overweight women is $\sim 18 \%$ (11), we calculated that 108 women in each study group would be required to demonstrate a 67\% GDM reduction in the myo-inositol group with a statistical power of $80 \%(\alpha=0.05)$. Statistical analysis was carried out with SPSS statistical package version 17 (SPSS, Chicago, IL). Data are expressed as mean (SD) for continuous variables and as percentages for categorical variables. The means of independent groups were compared using Student $t$ test after checking for normal distribution. For comparison of frequencies, Pearson $X^{2}$ test was used or, in the case of small frequencies, the Fisher exact test. A logistic regression analysis was performed in order to assess odds ratios (ORs) adjusted for treatment with myo-inositol and recognized risk factors for GDM, such as pre-pregnancy BMI, parity, maternal age, family history of $\mathrm{DM}$ and weight gain at OGTT. A value of $p \leq 0.05$ was considered statistically significant.
Table 1. General characteristics of the study groups at the I trimester.

\begin{tabular}{lccc}
\hline & $\begin{array}{c}\text { Myo-inositol } \\
(n=95)\end{array}$ & $\begin{array}{c}\text { Placebo } \\
(n=102)\end{array}$ & $p$ value \\
\hline Age (years) & $32.1 \pm 4.8$ & $32.7 \pm 5.3$ & 0.4 \\
Nulliparous $(n)$ & $49(51.6 \%)$ & $54(52.9 \%)$ & 0.8 \\
Pre-pregnancy BMI & $26.9 \pm 1.3$ & $27.1 \pm 1.3$ & 0.4 \\
Family history of DM II $(n)$ & $46(48.4 \%)$ & $34(33.3 \%)$ & $<0.001$ \\
GA at OGTT (days) & $181.2 \pm 9.9$ & $180.2 \pm 12.2$ & 0.5 \\
Fasting glucose (mg/dl) & $81.09 \pm 8.03$ & $78.63 \pm 6.15$ & 0.3 \\
Fasting insulin (MU/ml) & $10.77 \pm 7.99$ & $9.96 \pm 11.70$ & 0.8 \\
HOMA-IR & $2.18 \pm 1.69$ & $1.60 \pm 1.08$ & 0.2 \\
\hline
\end{tabular}

GA, gestational age; OGTT, oral glucose tolerance test; MU, micro nternational units/ml; HOMA-IR, homeostasis model assessment of insulin resistance.

Table 2. OGTT glucose values and incidence of gestational diabetes.

\begin{tabular}{lccc}
\hline & $\begin{array}{c}\text { Myo-inositol } \\
(n=95)\end{array}$ & $\begin{array}{c}\text { Placebo } \\
(n=102)\end{array}$ & $p$ value \\
\hline Glycemia T0 $(\mathrm{mg} / \mathrm{dl})$ & $80.5 \pm 7.3$ & $82.5 \pm 8.6$ & 0.09 \\
Glycemia T60 $^{\prime}(\mathrm{mg} / \mathrm{dl})$ & $128.5 \pm 30.2$ & $133.4 \pm 32.2$ & 0.3 \\
Glycemia T120' $(\mathrm{mg} / \mathrm{dl})$ & $106.6 \pm 28.0$ & $113.4 \pm 27.4$ & 0.07 \\
GDM rate & $11.6 \%(n=11)$ & $27.4 \%(n=28)$ & 0.004 \\
\hline
\end{tabular}

\section{Results}

In the myo-inositol group, there was one mid-trimester miscarriage, five women delivered in other hospitals (thus, it was impossible to obtain their records) and two women abandoned the trial, not attending the OGTT evaluation. Furthermore, there were seven dropouts, principally because women were negatively influenced by their relatives and family doctor's advice, leaving 95 women for the analysis. No women complained for side effects of the drug. In the placebo group, there was one mid-trimester miscarriage, two women abandoned the trial, not attending the OGTT evaluation, and five delivered in other hospitals, leaving 102 women for the analysis. The two groups were comparable for maternal age, pre-pregnancy BMI, percentage of nulliparous women, glycemia, insulinemia and HOMA-IR at first trimester of pregnancy (Table 1). Women with family history of type 2 DM were more represented in the myo-inositol group (Table 1). The incidence of GDM, the main outcome measure, was significantly reduced in the myo-inositol group $(n=11$, $11.6 \%)$ compared with the placebo group $(n=28,27.4 \%)$ $(p=0.004)$. After adjustment for confounding factors such as: maternal age, pre-pregnancy BMI, weight increase at OGTT, parity and family history for type $2 \mathrm{DM}$, only myo-inositol treatment was associated with a significant reduction of GDM development risk [OR 0.33 (95\% CI 0.15-0.70)]. No significant difference in glycemia at each steps of OGTT values between the two groups was highlighted (Table 2); as well as in weight gain at the OGTT (Table 3). In the placebo group, all women with GDM were treated by diet, but there were four who needed insulin; in the myo-inositol group, only two women with GDM needed insulin (Table 3). When considering secondary outcomes there was no difference between groups in the percentage of cesarean sections (both electives that emergency, to maternal and/or fetal causes), birth weight, 
Table 3. Secondary outcomes in both groups.

\begin{tabular}{lccc}
\hline & $\begin{array}{c}\text { Myo-inositol } n=95 \\
(11 \mathrm{GDM})\end{array}$ & $\begin{array}{c}\text { Placebo } n=102 \\
(28 \mathrm{GDM})\end{array}$ & $p$ value \\
\hline Weight gain at OGTT (kg) & $6.2 \pm 3.2$ & $7.5 \pm 4.0$ & 0.07 \\
GA at delivery (days) & $273.5 \pm 9.4$ & $272.4 \pm 10.4$ & 0.4 \\
Birthweight (g) & $3164.6 \pm 462.0$ & $3221.6 \pm 508.2$ & 0.4 \\
Cesarean section & $38(40 \%)$ & $49(48 \%)$ & 0.3 \\
CS in emergency & $7(7.4 \%)$ & $16(15.7 \%)$ & 0.1 \\
Macrosomia & $1(1 \%)$ & $5(4.9 \%)$ & 0.2 \\
Pre-term delivery & $2(2.1 \%)$ & $8(7.8 \%)$ & 0.3 \\
Gestational hypertension & $1(1 \%)$ & $4(3.9 \%)$ & 0.2 \\
Transferred to NICU & $1(1 \%)$ & $1(1 \%)$ & 0.9 \\
Shoulder dystocia & 0 & $1(1 \%)$ & 0.9 \\
Insulin treatment & $2(18.2 \%)^{*}$ & $4(14.3 \%)^{*}$ & $0.85^{*}$ \\
Neonatal hypoglycemia & $0 *$ & $1(3.6 \%)^{*}$ & $0.62 *$ \\
\hline
\end{tabular}

CS, cesarean section; NICU, neonatal intensive care unit.

*Statistical analysis considers only GDM population.

gestational age at delivery, macrosomia, PIH, shoulder dystocia, neonatal hypoglycemia and babies transferred to NICU (Table 3). At the logistic regression analysis, only the treatment with myo-inositol $(p=0.03)$ was independently associated to the onset of GDM.

\section{Discussion}

This study shows that myo-inositol intake, since early stages of pregnancy, in overweight women reduces the incidence of GDM, confirming the positive effect of this molecule already highlighted in previous reports [5,6]. Indeed, myo-inositol supplementation reduced the occurrence of GDM in women at risk for a positive family history of diabetes mellitus $(6 \%$ versus $15.3 \%$ ) [6]; moreover, the occurrence of GDM in obese pregnant women was dramatically reduced from $33.6 \%$ to $14 \%$ in a study recently published by our research group [5]. Several evidences demonstrate that maternal high pre-pregnancy BMI has a negative impact on pregnancies outcomes, with regard to the onset of GDM, hypertensive disorders of pregnancy and other adverse fetal outcomes [11,12]. This is probably caused by changes in the impairment in glucose and lipid metabolism probably already altered in overweight women. Pregnancy is physiologically characterized by hyperinsulinemia and insulin resistance [13]. During the third trimester, insulin sensitivity is reduced up to 50-70\% less than the pre-pregnancy status [14]. Insulin resistance is a common condition in overweight/obese subjects, because of the endocrine activity of adipokynes produced by visceral adipose tissue. It is common knowledge that the increased visceral adipose tissue regulates the glucose homeostasis, the energy metabolism, the immune system the coagulation and the blood pressure [15]. Indeed, abdominal visceral adiposity seems to have the potential to play a greater role than the whole body adiposity in high weight-related pregnancy complications. GDM is almost a worldwide epidemic; it could be expected that the prevalence of GDM will continue to undergo an increase because of both the introduction of new risk factors (often recognized in the general population) and the lowering of thresholds (just a value outside the cut off is enough for the GDM diagnosis) requested for the diagnosis. GDM is associated with increased rates of fetal morbidity and mortality, both during the pregnancy and in the postnatal life [16]. Moreover, women with GDM and their infants are at increased risk for diabetes mellitus and metabolic dysfunction later in life [17]; thus, strategies to prevent such condition would be preferable with respect to its treatment. Current approaches to prevent GDM have focused on lifestyle interventions such as diet and physical activity [18]. However, the authors of the last Cochrane review concluded that based on the data currently available, there are no clear difference in the risk of developing GDM for women receiving a combined diet and exercise intervention compared to women receiving no intervention. Recently, some supplements are emerging as additional options; one of these is myo-inositol, which is an insulin sensitizer substance able to improve glucose homeostasis. Its mechanism of action is previously widely described [19]. However, it is remarkable that in spite of different pre-gestational BMI mean values: 22.8 [5], 33.8 [6] and 26.9 in this study, myo-inositol intake determines the same effect, with similar reduction in GDM rate and consequently similar $\mathrm{OR}(0.35,0.34,0.33$, respectively). We argue that this data cannot be occasional, whether the same reduction in GDM rate is obtained from group of women with so different pre-gestational BMI. These results are certainly associated with the insulin resistance reduction induced by myo-inositol, clearly demonstrated in the study on obese women [6]. Despite the reduction in GDM rate, no improvement of clinical outcomes, such as macrosomia and other co-morbidity related to GDM (hypertensive disorders, shoulder dystocia and pre-term delivery), have been demonstrated in this study. This could simply depend by the lack of power respect with previous outcomes. Indeed, the prevalence of macrosomia or shoulder dystocia is very low. Thus, further studies are needed with much larger sample of women to confirm these results and to establish whether a dietary supplement such as myo-inositol, may reduce not only GDM rate, but also its related fearful complications.

\section{Declaration of interest}

The authors report no declarations of interest.

\section{References}

1. Gestational diabetes. ACOG Practice Bulletin No. 30. American College of Obstetricians and Gynecologists. Obstet Gynecol 2001; 98:525-38.

2. Metzger BE, Lowe LP, Dyer AR, et al. Hyperglycemia and adverse pregnancy outcomes. N Engl J Med 2008;358:1991-2002.

3. Metzger BE, Gabbe SG, Persson B, et al. International association of diabetes and pregnancy study groups recommendations on the diagnosis and classification of hyperglycemia in pregnancy. Diabetes Care 2010;33:676-82.

4. American Diabetes Association. Diagnosis and classification of diabetes mellitus. Diabetes Care 2011;34:S62-9.

5. D'Anna RD, Benedetto A, Scilipoti A, et al. Myo-inositol supplementation for the prevention of gestational diabetes in obese pregnant women. Obstet Gynecol 2015;126:310-15.

6. D’Anna R, Scilipoti A, Giordano D, et al. Myo-Inositol supplementation and onset of gestational diabetes mellitus in pregnant women with a family history of type 2 diabetes: a prospective, randomized, placebo-controlled study. Diabetes Care 2013;36: 854-7.

7. Corrado F, D’Anna R, Di Vieste G, et al. The effect of myoinositol supplementation on insulin resistance in patients with gestational diabetes. Diabetes Med 2011;28:972-5.

8. Genazzani AD, Lanzoni C, Ricchieri F, Jasonni VM. Myo-inositol administration positively affects hyperinsulinemia and hormonal 
parameters in overweight patients with polycystic ovary syndrome. Gynecol Endocrinol 2008;24:139-44.

9. Croze ML, Soulage CO. Potential role and therapeutic interests of myo-inositol in metabolic diseases. Biochimie 2013;95:1811-27.

10. Corrado F, Pintaudi BD, Vieste G, et al. Italian risk factor-based screening for gestational diabetes. J Matern Fetal Neonatal Med 2014;27:1445-8.

11. Zhang Y, Wang ZL, Liu B, Cai J. Pregnancy outcome of overweight and obese Chinese women with gestational diabetes. J Obstet Gynaecol 2014;34:662-5.

12. Pintaudi B, Di Vieste G, Corrado F, et al. Improvement of selective screening strategy for gestational diabetes through a more accurate definition of high risk groups. Eur J Endocrinol 2014;170:87-93.

13. Hadden DR, McLaughlin C. Normal and abnormal maternal metabolism during pregnancy. Semin Fetal Neonatal Med 2009; 14:66-71.
14. Catalano PM, Tyzbir ED, Roman NM, et al. Longitudinal changes in insulin release and insulin resistance in nonobese pregnant women. Am J Obstet Gynecol 1991;165:1667-72.

15. Wajchemberg BL. Subcutaneous and visceral adipose tissue: their relation to the metabolic syndrome. Endocr Rev 2000;21:697-738.

16. Devay RX. Gestational diabetes mellitus: a review from 2004. Curr Diabetes Rev 2005;1:203-13.

17. Shah BR, Retnakaran R, Booth GL. Increased risk of cardiovascular disease in young women following gestational diabetes mellitus. Diabetes Care 2008;31:1668-9.

18. Bain E, Crane M, Tieu J, et al. Diet and exercise interventions for preventing gestational diabetes mellitus. Cochrane Database Syst Rev 2015;4:CD010443.

19. Facchinetti F, Dante G, Petrella E, Neri I. Dietary interventions, lifestyle changes and dietary supplements in preventing gestational diabetes mellitus: a literature review. Obstet Gynecol Survey 2014; 69:669-80. 